\title{
Assessment of the effect of continuous theta burst stimulation of the motor cortex on manual dexterity in non-human primates in a direct comparison with invasive intracortical pharmacological inactivation
}

\author{
Camille Roux ｜ Mélanie Kaeser ｜ Julie Savidan ｜ Michela Fregosi | Eric M. Rouiller \\ Eric Schmidlin
}

Section of Medicine, Department of

Neurosciences and Movement

Sciences, Faculty of Science and

Medicine, University of Fribourg, Fribourg,

Switzerland

\section{Correspondence}

Eric Schmidlin, Section of Medicine,

Department of Neurosciences and

Movement Sciences, Faculty of Science

and Medicine, University of Fribourg,

Chemin du Musée 5, CH-1700 Fribourg,

Switzerland.

Email: eric.schmidlin@unifr.ch

Funding information

Schweizerischer Nationalfonds zur

Förderung der Wissenschaftlichen

Forschung, Grant/Award Number:

310000-110005, 31-61857.00,

31003A-132465 and PZ00P3_142258

\begin{abstract}
Non-invasive reversible perturbation techniques of brain output such as continuous theta burst stimulation (cTBS), commonly used to modulate cortical excitability in humans, allow investigation of possible roles in functional recovery played by distinct intact cortical areas following stroke. To evaluate the potential of cTBS, the behavioural effects of this non-invasive transient perturbation of the hand representation of the primary motor cortex (M1) in non-human primates (two adult macaques) were compared with an invasive focal transient inactivation based on intracortical microinfusion of GABA-A agonist muscimol. The effects on the contralateral arm produced by cTBS or muscimol were directly compared based on a manual dexterity task performed by the monkeys, the "reach and grasp" drawer task, allowing quantitative assessment of the grip force produced between the thumb and index finger and exerted on the drawer's knob. cTBS only induced modest to moderate behavioural effects, with substantial variability on manual dexterity whereas the intracortical muscimol microinfusion completely impaired manual dexterity, producing a strong and clear cortical inhibition of the M1 hand area. In contrast, cTBS induced mixed inhibitory and facilitatory/ excitatory perturbations of M1, though with predominant inhibition. Although cTBS impacted on manual dexterity, its effects appear too limited and variable in order to use it as a reliable proof of cortical vicariation mechanism (cortical area replacing another one) underlying functional recovery following a cortical lesion in the motor control domain, in contrast to potent pharmacological block generated by muscimol infusion, whose application is though limited to an animal model such as non-human primate.
\end{abstract}

\footnotetext{
Abbreviations: 1DI, one dorsal interosseous muscle; AbPB, abductor pollicis brevis muscle; cTBS, continuous theta burst stimulation; EDC, extensor digitorum communis muscle; EMG, electromyography; FCU, flexor carpi ulnaris muscle; FDS, flexor digitorum superficialis muscle; ICMS, intracortical microstimulation; M1, primary motor cortex; Mk, monkey; MSO, maximal stimulation output (of TMS); N, Newtons; PL, palmaris longus muscle; PMv, ventral premotor cortex; R0, resistance level 0 (0 Newtons, negligible resistance to drawer opening); R5, resistance level 5 (2.25 Newtons resistance to drawer opening); TMS, transcranial magnetic stimulation.
}

Roux and Kaeser are shared first authorship.

Edited by Yoland Smith. Reviewed by Allan Smith.

All peer review communications can be found with the online version of the article. 


\section{KE Y W O R D S}

continuous theta burst stimulation, cortical plasticity, manual dexterity, motor cortex,

non-human primate, transient inactivation

\section{$1 \mid$ INTRODUCTION}

Despite the absence of clear signs of regeneration after cortical lesion, the central nervous system of adult mammals is capable of a certain level of spontaneous functional recovery, depending on the extent and the localization of the lesion. In patients suffering from stroke affecting motor control, changes of cortico-cortical interactions have been observed, within the ipsilesional hemisphere (Swayne, Rothwell, Ward, \& Greenwood, 2008), affecting the corticospinal tract (Schaechter et al., 2009) and/or even involving the contralesional hemisphere (Bestmann et al., 2010). The exact mechanisms behind this recovery are still a matter of debate. In this context, non-human primate (NHP) models are valuable tools to study mechanisms of cortical plasticity following localized specific lesion of the motor cortex, such as interhemispheric changes of cellular density in premotor areas (Contestabile et al., 2018), in rewiring of cortico-cortical connections (Dancause et al., 2005) or vicariation (Hoogewoud et al., 2013; Liu \& Rouiller, 1999; Murata et al., 2015). Theories of functional recovery distinguished redundancy from vicariation (see Finger, 2010 for review; see also Slavin, Lawrence, \& Stein, 1998). Redundancy refers to the presence of duplicate or back-up similar cortical areas which can take over the function lost after damage of a specific brain area. In vicariation, in contrast, the lost function is taken over by a brain area with an originally different function from the damaged area (e.g., PM "replacing" M1 to some extent when M1 is lesioned).

Manual dexterity in non-human primates is an adequate behavioural readout to study the effects of a motor cortex lesion (Darling et al., 2016; Morecraft et al., 2016; Schmidlin et al., 2011; Wyss et al., 2013), as well as the subsequent functional recovery due to cortical plasticity. Indeed, fine motor control such as precision grip involved in manual dexterity is a characteristic of primates (Lawrence, Porter, \& Redman, 1985; Lemon, 2008b; Lemon \& Griffiths, 2005; Rouse \& Schieber, 2016). Manual dexterity results from the elaboration of complex motor output to spinal motoneurons computed by interactions between premotor areas such as the premotor cortex (PM), supplementary motor area (SMA) and the primary motor cortex (M1) (Lemon, 2008a; Schmidlin, Brochier, Maier, Kirkwood, \& Lemon, 2008). An invasive inactivation study showed the preponderant role played by M1 in the transmission of adequate cortical output signals to the spinal cord (Schmidlin et al., 2008).

Following a lesion of a motor cortical area, incomplete spontaneous functional recovery takes place, based on a vicarious principle that other motor areas originally with different functions and not impacted by the lesion may take over the lost motor functions, at least to some extent. The experimental demonstration of such mechanism of recovery from the cortical lesion requires inactivating, ideally transiently, the putative cortical areas which took over part of the lost function, in order to reproduce the more extensive deficits observed in the acute phase post-lesion. In non-human primates, such experimental cortical inactivation can be produced invasively using a reversible pharmacological block, with intracortical infusion of the GABA-A agonist muscimol or the local anaesthetic lidocaine for instance (Hoogewoud et al., 2013; Kermadi, Liu, Tempini, \& Rouiller, 1997; Liu \& Rouiller, 1999; Rouiller, Yu, \& Tempini, 1997). In human subjects (e.g., stroke patients), only a non-invasive experimental demonstration of vicariation can be envisaged based on the perturbation of the putative cortical area contributing to the functional recovery, using for instance transcranial magnetic stimulation (TMS) in the form of continuous theta burst stimulation (cTBS; e.g., Goldsworthy, Pitcher, \& Ridding, 2012; Suppa et al., 2016). In order to evaluate the potential of the cTBS perturbation to study the post-lesion cortical reorganization as regard to the currently used pharmacological inactivation, we directly paralleled these two methods of modulating a motor cortical area, by assessing the behavioural impact of either muscimol infusion in M1 or of cTBS applied over M1 in the same individual non-human primate, performing the same controlled manual dexterity task. As the strong effect of pharmacological inactivation of the motor cortex on manual dexterity is well documented and reproducible (Hoogewoud et al., 2013; Kermadi et al., 1997; Liu \& Rouiller, 1999; Rouiller et al., 1997), muscimol was infused only once in each of the two current experimental monkeys (to preserve their motor cortex for the long-term protocol), whereas emphasis was put here on the largely unknown cTBS perturbation procedure in macaques, which was repeated in several consecutive sessions to quantitatively assess its effect and reproducibility.

To this aim, two adult macaque monkeys were first subjected to repeated daily sessions of cortical perturbation by applying cTBS, as currently used in healthy human subjects (Georgiev et al., 2016; Huang, Edwards, Rounis, Bhatia, \& Rothwell, 2005; Nyffeler, Cazzoli, Hess, \& Muri, 2009; Suppa et al., 2016), based on a particular paradigm of stimulation aiming to decrease the amplitude of motor evoked potentials in the finger muscles during a time period up to 40 min (Goldsworthy et al., 2012). Second, it was followed 
by a single invasive session of intracortical microinfusions of the GABA-A agonist muscimol in the hand representation of M1 (Brochier, Boudreau, Pare, \& Smith, 1999; Hoogewoud et al., 2013; Kermadi et al., 1997; Liu \& Rouiller, 1999; Schmidlin et al., 2008). These two methods have been applied in each of two adult macaques performing a manual dexterity task with their contralateral hand, challenging fine motor control and precision grip, the "reach and grasp" drawer task (Schmidlin et al., 2011). We have tested the hypothesis that both cortical perturbation methods induced largely comparable behavioural effects on manual dexterity, though to a different extent, in the form of a reduction of performance and ability to produce force between the thumb and index finger. The "reach and grasp" drawer task was chosen to address this issue, as it is a manual dexterity task yielding quantitative data on force, a motor parameter more sensitive than an assessment limited to a behavioural score.

\section{MATERIALS AND METHODS}

\section{1 | Macaque monkeys}

Two adult purpose-bred macaque monkeys (Macaca fascicularis), Mk-CA (10 years old, weight $5.9 \mathrm{~kg}$; female) and Mk-DG (11 years old, weight $7.9 \mathrm{~kg}$; male), enrolled in other main projects related to permanent lesion of M1, were used occasionally in the present side-project aimed at comparing the two reversible cortical inactivation/perturbation methods. The number of inactivation/perturbation sessions was kept to a minimum, to avoid motor cortex damage which may compromise the main injury studies. Complementary behavioural data from Mk-CA and additional histological data from MkDG were reported in recent publications (Kaeser et al., 2014; Savidan, Kaeser, Belhaj-Saif, Schmidlin, \& Rouiller, 2017). All procedures, including purchase of monkeys from a recognized supplier and detention conditions at the University of Fribourg, were conducted according to the guidelines edited by the Swiss federal law on animal protection, reviewed and approved by the local ethical committee ("Commission cantonale de surveillance de l'expérimentation animale"), and finally authorized by the competent veterinary authorities (cantonal and federal), under the license number 2014_42_FR.

\subsection{General survey of the experimental protocol}

The animals were first trained to perform the "reach and grasp" drawer task until they reached a plateau of behavioural performance after several months (Kaeser et al., 2014; Schmidlin et al., 2011). For the cTBS experimental sessions (Figure 1a), the animals were habituated, while sitting in the primate chair in a comfortable position, to have the head fixed using a heat malleable personalized plastic helmet, adapted to the head shape of each monkey (De Luna, Mohamed Mustafar, \& Rainer, 2014). This head fixation procedure (Figure 1a, rightmost panel) allowed the positioning of the "figure of eight" pediatric coil (Figure 1a) directly at the surface of the head tangentially to the frontal lobe to target M1.

A tecapeek chronic chamber was surgically implanted in order to allow precise mapping of the hand representation in M1 and PMv (ventral premotor cortex) using intracortical microstimulation (ICMS; Figure 1b) and then micro-infuse the GABAergic agonist muscimol in M1, following a procedure reported earlier (Schmidlin et al., 2008). The intracortical muscimol microinfusion was performed while the head was stabilized using an external Perspex frontal plate $(30 \mathrm{~cm}$ by $20 \mathrm{~cm})$ with a nearly oval hole in the middle $(6 \mathrm{~cm}$ horizontal by $9 \mathrm{~cm}$ vertical), in which the monkey inserted the snout. The frontal plate was then fixed to the set-up, thus preventing large head movements when muscimol was infused using a Hamilton Needle Assy (51315-02) of the same diameter as the ICMS electrode. The infusion needle was prolonged by a flexible tubing attached to a Hamilton syringe. The infusion needle was lowered into M1 via the tecapeek grid (Figure 1b), at depths previously determined based on ICMS.

The temporal sequence of the overall experiment in the two monkeys is illustrated in Figure 2a. In both monkeys, the repeated cTBS daily inactivation sessions took place when the two hemispheres were still intact. In Mk-CA, overall four cTBS inactivation sessions were performed, as well as three sham-cTBS sessions within a time window of 52 days (see Figure $2 \mathrm{a}$, including the precise time intervals between the consecutive sessions). One of the cTBS session consisted in a double cTBS stimulation paradigm. About 4 months after, the cTBS protocol was completed in Mk-CA, repeated ICMS sessions were conducted during 8 weeks in order to electrophysiologically map M1. When the hand area in the left M1 was identified, a muscimol inactivation session was conducted, $24 \mathrm{hr}$ after a brief ICMS session in which the final sites and their depths for infusion were confirmed (Figure 2a). Mk-CA fully recovered from the muscimol inactivation the day after (Figure 2a). Later, ICMS was repeated, followed by the permanent lesion of the left M1, for the purpose of the separated main M1 injury project. In Mk-DG, when the two hemispheres were still intact, cTBS inactivation sessions were conducted in the right $\mathrm{M} 1$, including one sham session, within a time window of 3 days (Figure $2 \mathrm{a}$ ). Later, the opposite left M1 was mapped and permanently injured for the purpose of another study (Savidan et al., 2017). Finally, 4 months later, the intact right M1 was mapped with ICMS before conducting a muscimol inactivation session (Figure 2a), with however an overdose of muscimol (see below).

Once the transient perturbation experiments (muscimol and cTBS) were completed, chronic electromyographic (EMG) electrodes were surgically implanted in Mk-CA at the muscular surface of forelimb muscles directly involved in 
(a)

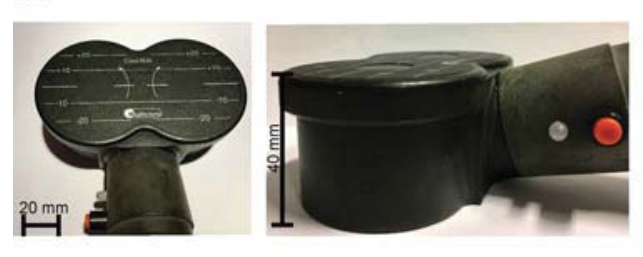

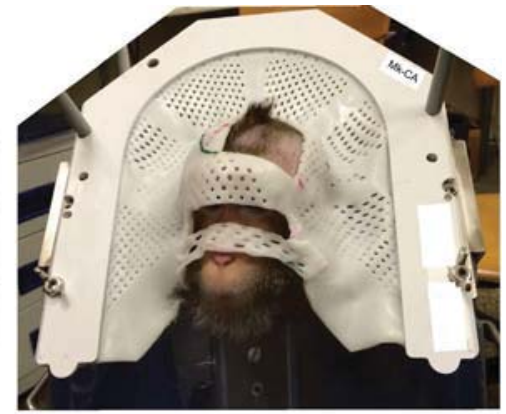

(b)

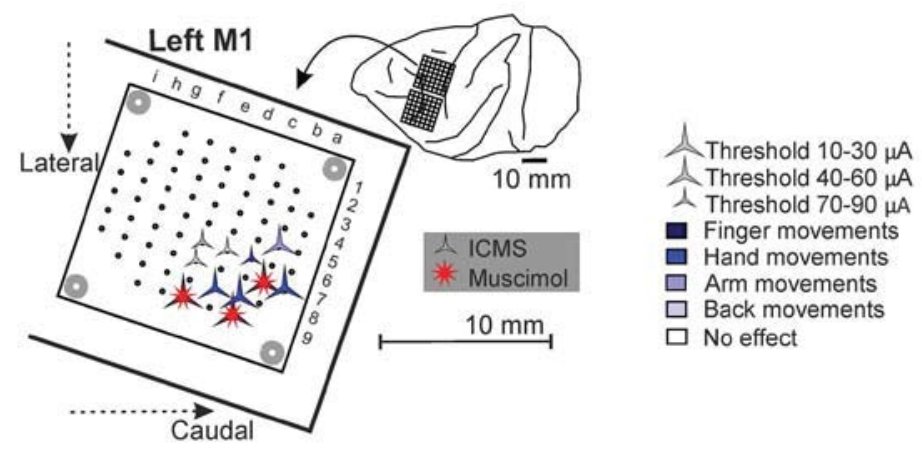

(c)

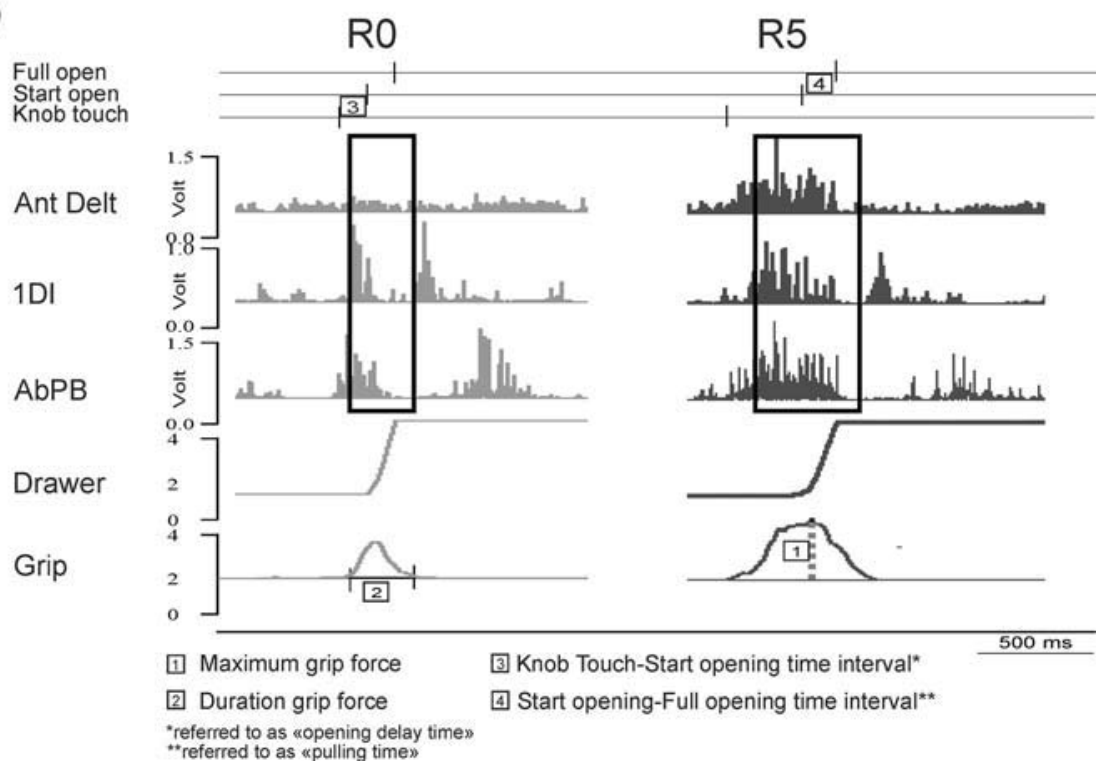

F IG URE 1 (a) On the left, two pictures of the paediatric coil (form of eight) used to apply cTBS above M1 in macaque monkeys. On the right, view of Mk-CA in its primate chair, wearing the home-made malleable personalized plastic helmet (modified from De Luna et al., 2014) to stabilize the monkey's head for the cTBS sessions. Note the presence of a hole in the helmet above the left M1, allowing the application of cTBS to that target. (b) The top right inset shows the left hemisphere of Mk-CA, with the location of the two grid chronic chambers above M1 and PMv, respectively. Relevant for this study is the grid over M1 represented at higher magnification (curved arraow), displaying the ICMS map of the left M1 in Mk-CA, with sites of muscimol infusion in red. The sites of microinfusion of muscimol were estimated at 3, 4 and $5 \mathrm{~mm}$ under the dura-mater encephali. The ICMS map shows the body territories (hand, etc) as well as the ICMS threshold intensities to observe the corresponding movement of the contralateral forelimb. (c) Behavioural recordings of two representative trials performed by Mk-CA during a "reach and grasp" drawer task at two different levels of resistance to the opening (left panel: relative resistance $=0$ and right panel: relative resistance $=5$ ). The figure illustrates the motor parameters which have been quantified to assess the consequences of motor cortical perturbation: amplitude of maximal grip force (1); duration of grip force exerted on the drawer's knob (2); time delay to initiate the opening of the drawer (3); and the pulling time corresponding to the time interval to completely open the drawer (4). This graph also shows the involvement of intrinsic and extrinsic hand muscles (EMG activity) as well as the anterior deltoid during the performance of the task and the increased grip force needed when the resistance to the opening was increased from R0 to R5 (EMG activity within the two rectangles) 


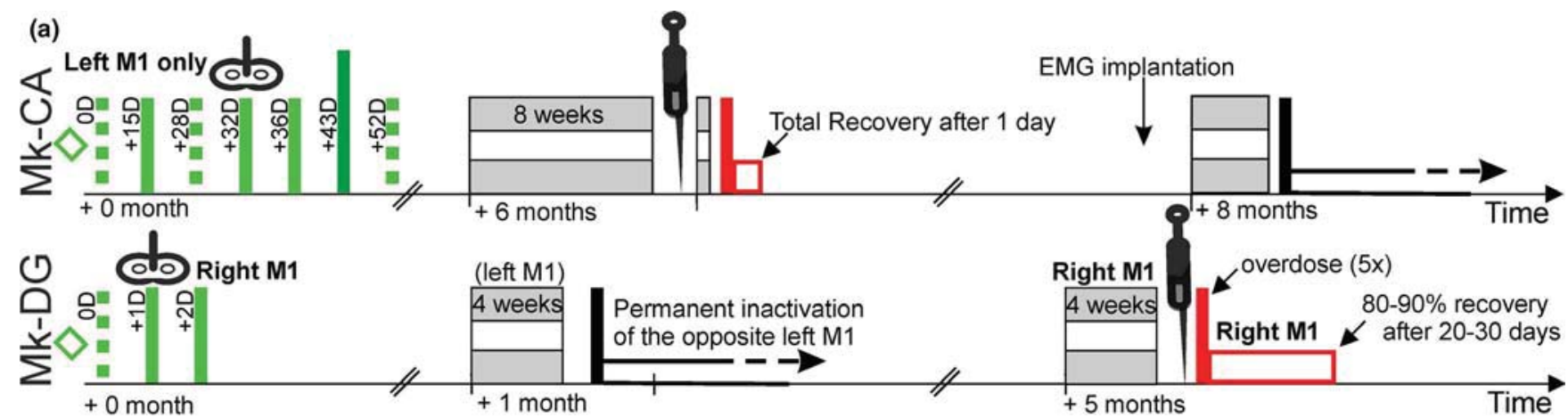

$\checkmark$ Single pulses TMS (mapping) m = $=$ : Sham-cTBS cTBS

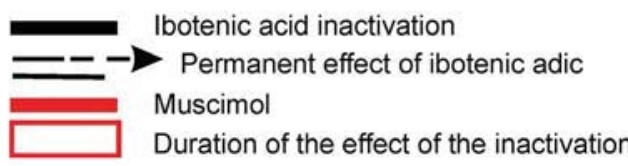

electrophysiol. mapping ICMS

double cTBS

Post-perturbation

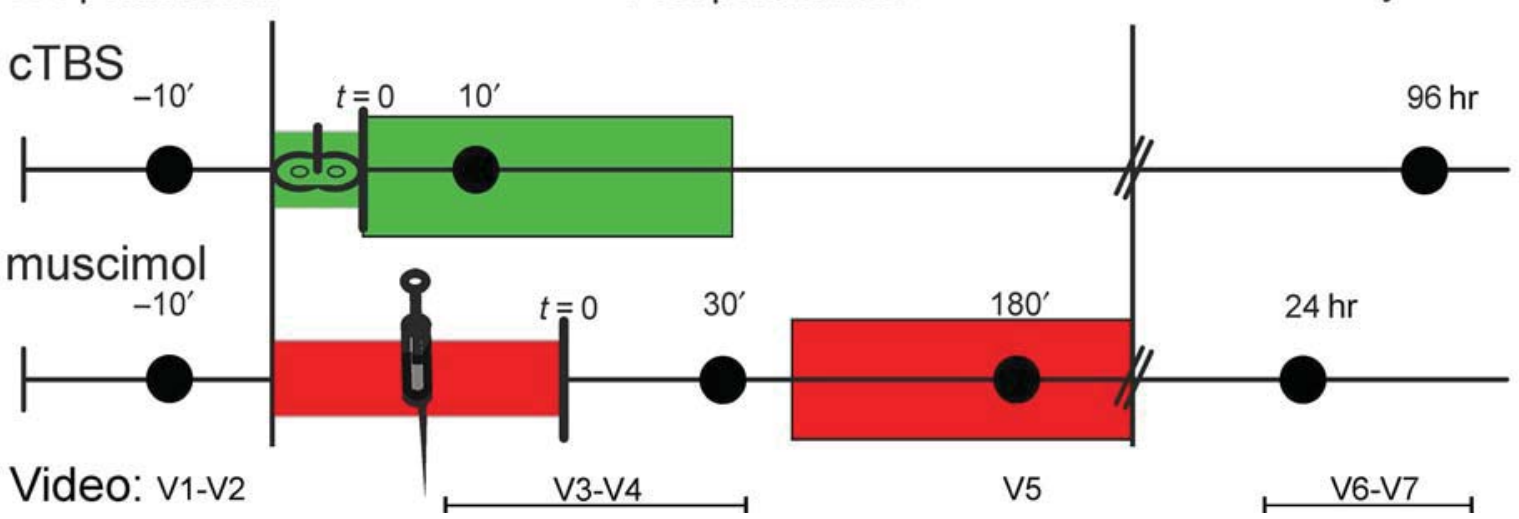

F IG URE 2 (a) Schematic representation of the temporal sequence of the different experimental steps performed in each of the two monkeys Mk-CA (upper row) and Mk-DG (lower row). The sequence of the two transient perturbations/inactivations is indicated in green for the cTBS and in red for the muscimol microinfusion. Note the presence of intermingled sham-cTBS sessions. The eight shape coil is for cTBS session period, while the syringe indicates the time point of the muscimol inactivation. The interval between the consecutive cTBS sessions is indicated in days (for instance 0D, 15D, 28D, etc), starting at day zero for the first cTBS daily session. Note the presence before (green diamond) of single pulse TMS to map M1 (see Methods 2). In addition, ICMS mapping of M1 preceding the muscimol inactivation sessions is indicated, as well as before the permanent lesion pertinent for the main separate projects, shown here for general information purposes. In Mk-CA, all interventions took place in the left M1. In Mk-DG, the intervention pertinent to the present study occurred in the right M1, except an ibotenic acid permanent lesion of the opposite left M1. The ibotenic acid lesions induced permanent deficit (dashed horizontal black lines), whereas the recovery from the muscimol session is indicated with the red rectangles. The time scale (in days/months) is interrupted in order to maximize the resolution of the figure. (b) Schematic comparison cTBS (green) versus muscimol (red) of an individual perturbation/inactivation session, showing the precise timing of behavioural testing (black spheres). Rightmost coloured area corresponds to the maximal expected effect of each perturbation/inactivation technique, $10 \mathrm{~min}$ after end of cTBS and $40 \mathrm{~min}$ after the last muscimol microinfusion. Black discs correspond to the behavioural tests to assess manual dexterity before, immediately after and in the functional recovery period. The time points of the seven videos are also indicated

the "reach and grasp" drawer task, such as Abductor Pollicis Brevis (AbPB) and one dorsal interosseous (1DI), using tungsten microwires. Therefore, the EMG recordings were not available at the time of cTBS and muscimol sessions, but only at later phases of the overall research project (involving permanent lesion of M1). These EMG data in Mk-CA were nevertheless pertinent to better characterize the behavioural "reach and grasp" drawer task from which the quantitative inactivation data were derived (Figure 1c).

\section{3 | cTBS stimulation and TMS identification of M1 hand representation}

To functionally identify the left M1 hand area before the cTBS sessions (Figure 2a), a physiological mapping of M1 using TMS single pulses was conducted in Mk-CA in two steps. A first in-depth TMS session was performed in order to establish an extensive mapping of M1 (at a time point of minus 8 days with reference to the day zero in the time line 
of Figure 2a: diamond symbol). From this single pulse TMS mapping, a mark was made on the surface of the skin corresponding to the centre of the coil where single joint movements in the contralateral hand (mostly thumb and index finger) were observed at lowest threshold of stimulation. On the day of each individual cTBS session, a rapid TMS single pulse mapping was performed in order to confirm the optimal coil position above the left M1. In Mk-DG, the same TMS mapping preceding the cTBS sessions was conducted in two steps as well.

The cTBS for transient perturbation consisted in 200 trains of three pulses each, separated by $33.3 \mathrm{~ms}$, and an inter-train time interval of $167 \mathrm{~ms}$, for a total duration of $30 \mathrm{~s}$ (Goldsworthy et al., 2012). The stimulation intensity was set at $80 \%$ of the lowest stimulation intensity needed to elicit single joint movement and was in the range of $37 \%-44 \%$ of the maximal stimulation output (MSO) of the TMS system (MagVenture Pro X100, Coil B-35 Pediatric; see Figure 1a). The threshold was defined as the intensity eliciting a response in five out of 10 pulses. One of the cTBS experiments conducted in Mk-CA involved two consecutive cTBS, 15 min apart, to evaluate possible increase of cortical perturbation consequent to double cTBS (Table 1, Mk-CA (D)). Finally, at the end of the cTBS session, the adequate positioning of the coil was checked by repeating the rapid single pulse TMS mapping (except in the second cTBS session of Mk-DG: see Table 1, symbol \&).

\section{4 | Muscimol inactivation and ICMS identification of M1}

Under anaesthesia maintained by endotracheal inhalation of sevoflurane 2.5\%, combined with Propofol (0.1 $\mathrm{mg} \mathrm{kg}^{-1} \mathrm{~min}^{-1}$ ) intravenous infusion (Lanz et al., 2013), a rectangular Tecapeek chronic chamber was surgically implanted unilaterally above the rostral part of the central sulcus according to topographic coordinates (Shimazu, Maier, Cerri, Kirkwood, \& Lemon, 2004). The chronic chamber includes two squared tecapeek grids containing 49 holes each with a diameter of $500 \mu \mathrm{m}$ perpendicular to the surface (Figure 1b), allowing the trans-dural penetrations with tun microelectrodes (Savidan et al., 2017; Schmidlin et al., 2008). ICMS was performed under light sedation maintained with repeated intramuscular injections of ketamine $(1.25 \mathrm{mg} /$ $\mathrm{kg}$ ) combined with medetomidine $(0.07 \mathrm{mg} / \mathrm{kg})$. Just before the proper ICMS procedure, the anaesthesia was reversed with intramuscular injection of Atipamezole (200 mg/kg). After cleaning the grids, ICMS was applied to obtain consistent movements of the contralateral hand's fingers at low threshold and consisted in a train stimulation of six to nine pulses $(0.2 \mathrm{~ms}$ each) at maximum current intensity of $80 \mu \mathrm{A}$ (Andersen, Hagan, Phillips, \& Powell, 1975; Schmidlin, Wannier, Bloch, \& Rouiller, 2004). The hand representation of M1 was defined as the cortical territory in which single finger joint movements were elicited at $20 \mu \mathrm{A}$ or less

T A B LE 1 Survey of the individual cTBS and muscimol perturbation/inactivation sessions performed in Mk-CA and Mk-DG

\begin{tabular}{|c|c|c|c|c|c|c|c|c|c|}
\hline \multirow[b]{2}{*}{ Monkey } & \multirow[b]{2}{*}{ Inactivation } & \multicolumn{2}{|c|}{ Maximal grip force } & \multicolumn{2}{|c|}{ Duration of grip force } & \multicolumn{2}{|c|}{ Opening delay time } & \multicolumn{2}{|l|}{ Pulling time } \\
\hline & & R0 & R5 & R0 & R5 & R0 & R5 & R0 & R5 \\
\hline Mk-CA \# & cTBS & -3 & $-8^{* *}$ & $-6^{*}$ & -12 & +12 & -7 & -8 & $-14^{* *}$ \\
\hline Mk-CA & cTBS & $-7^{*}$ & $-6^{*}$ & -1 & -6 & +3 & -8 & -8 & -1 \\
\hline Mk-CA & cTBS & +2 & -3 & $-9^{*}$ & -1 & $-18^{*}$ & -6 & -7 & $+14^{* *}$ \\
\hline Mk-CA (D) & Double cTBS & $-11^{* *}$ & -3 & -17 & -13 & +5 & $-25^{*}$ & $-30^{* * *}$ & $-33^{* * *}$ \\
\hline Mk-DG & cTBS & $-11^{* *}$ & +2 & $-3^{*}$ & -4 & -10 & -3 & -2 & +6 \\
\hline Mk-DG \& & cTBS & -2 & -1 & $-3^{*}$ & -2 & -4 & -4 & -2 & -1 \\
\hline Mk-CA & $\begin{array}{r}\text { Muscimol } \\
(4.75 \mu \mathrm{g})\end{array}$ & Nil (-6) & Nil (Nil) & $\Delta\left(+76^{* * *}\right)$ & $\diamond(\diamond)$ & $\bullet\left(+195^{* * *}\right)$ & $\diamond(\diamond)$ & $\diamond\left(+40^{* * * *}\right)$ & $\diamond(\diamond)$ \\
\hline Mk-DG & $\begin{array}{c}\text { Muscimol } \\
(35 \mu \mathrm{g})\end{array}$ & $\operatorname{Nil}\left(-9^{* * *}\right)$ & Nil (Nil) & $\diamond\left(+280^{* * *}\right)$ & $\diamond(\diamond)$ & $\diamond\left(+220^{* * *}\right)$ & $\diamond(\diamond)$ & $\diamond\left(+179^{* * *}\right)$ & $\diamond(\diamond)$ \\
\hline
\end{tabular}

Note: There were four and two cTBS sessions in Mk-CA and Mk-DG, respectively. One muscimol session took place in each monkey (bottom two rows). Quantitative behavioural results derived from the cTBS and muscimol sessions obtained in the period of maximal expected effect of the corresponding transient perturbation in the two monkeys (two time points for muscimol: see below), expressed in per cent of change as compared to the values obtained in the pre-perturbation phase. Negative values express a decrease in per cent whereas positive values express an increase. Results obtained in the cTBS session when double cTBS was applied over the scalp within 15 min are identified with (D). The cTBS session illustrated in detail in Figures 3 and 4 is indicated by the \# symbol. In the bottom two rows, values in brackets express the data obtained in the early phase of muscimol inactivation (after $30 \mathrm{~min}$ ) whereas the data not in brackets are for the later phase (after 180 min). "Nil" indicates that the monkey was unable to perform the task, and therefore, no grip force could be measured. The same for diamonds as far as the temporal parameters are concerned. R0 and R5 are the two levels of resistance opposing the pulling of the drawer. For the two muscimol inactivation sessions, the total amount of muscimol infused in M1 is indicated in $\mu \mathrm{g}$. All behavioural effects are for the hand contralateral to the perturbed/inactivated M1.

*corresponds to a $p$-value $<0.05$.

***orresponds to a $p$-value $<0.01$.

$* * *$ corresponds to a $p$-value $<0.001$ 
(Schmidlin et al., 2008). The muscimol inactivation session took place $24 \mathrm{hr}$ later at most, in order to minimize the risk of pronounced changes of motor maps in the targeted M1 between the ICMS sessions and the muscimol inactivation.

Muscimol was infused in the monkey awake (head stabilized), in the hand representation of the left M1 in Mk-CA and of the right M1 in Mk-DG, respectively, using a Hamilton syringe and a 30-Gauge cannula. The concentration of muscimol in the saline solution was $1 \mu \mathrm{g} / \mu \mathrm{l}$ in Mk-CA and accidentally $5 \mu \mathrm{g} / \mu \mathrm{l}$ in Mk-DG (see also Savidan et al., 2017). In Mk-CA, muscimol was intracortically infused along three penetrations (Figure 1b), at three distinct sites (depths) previously identified with ICMS below the tecapeek grid (below the dura; nine infusion sites in total). At each site, a volume of $0.5-0.55 \mu \mathrm{l}$ of muscimol solution was infused in about $30 \mathrm{~s}$. The total amount of muscimol delivered in Mk-CA was $4.75 \mu \mathrm{g}$ (Table 1). The corresponding muscimol infusion parameters were recently reported for Mk-DG (Savidan et al., 2017), representing a total amount of $35 \mu \mathrm{g}$ (Table 1).

Due to the overdosed muscimol infusion in Mk-DG (Figure 2a), the inactivation was more potent and lasted longer than in Mk-CA, as it took in Mk-DG 20-30 days to observe a nearly complete recovery (80\%-90\%) of the contralateral (left) hand's manual dexterity, as assessed by the modified Brinkman board score and the Klüver board (Savidan et al., 2017).

\section{5 | Behaviour}

\subsection{1 | Reach and grasp drawer task}

Manual dexterity was tested using the "reach and grasp" drawer task (Kaeser et al., 2013; Schmidlin et al., 2011), a modified version of an earlier task used in the laboratory (Kazennikov et al., 1994). The animal performed a unimanual precision grip using opposition of the thumb and the index finger to grab the knob of a drawer containing a small food reward. Then, the monkey pulled the drawer against adjustable levels of resistance to the opening in order to grasp the food pellet using the same hand (see Videos S1-S7). Two levels of resistance were tested, levels 0 and 5, corresponding, respectively, to 0 and 2.25 Newtons $(\mathrm{N})$. This manual dexterity task required the specific involvement of both fingers pressing on the drawer's knob, as well as more proximal muscles such as the anterior part of the deltoid (see EMG recordings in Figure 1c). Every experimental session consisted in ten behavioural trials at each level of resistance, followed by the cortical perturbation, invasive (muscimol) or non-invasive (cTBS). Then, targeting the most efficient period of perturbation (cTBS: 10-30 min after cortical stimulation; muscimol: $30 \mathrm{~min}$ after the last cortical microinfusion), a second set of 30-40 trials was performed by the monkey following the same sequence of increased levels of resistance to the opening. During the muscimol inactivation experiment, an additional set of trials was added at a late time point $(180 \mathrm{~min})$ to assess the temporal dynamics of motor deficit due to the cortical inactivation. The typical temporal structure of the perturbation sessions (cTBS and muscimol) is illustrated in Figure 2b. All behavioural data presented in Table 1, as well as in Figures 3 and 4, are for the arm/hand contralateral to the perturbed/inactivated M1.

\subsection{2 | Behavioural quantification}

Four main motor parameters were quantified and compared. First, the "maximal grip force" exerted between the thumb and index finger on the drawer's knob, corresponding to the average of maximal values of grip force obtained in all trials of the same relative level of resistance. Second, the "grip force duration" was defined as the time interval between the onset and the offset of the grip force (Figure 1c). The onset and the offset of grip force were identified as the time points when the force in each trial crossed a reference of $10 \%$ of the force amplitude above baseline (resting force) in Newton (Figure 1c). Third, the "opening delay time" defined as the time interval between the knob touch and the onset of drawer pulling (time without constraint). Fourth, the "pulling time" defined as the time interval between pulling onset and the time point at which the drawer was fully open (time interval subjected to resistance constraint). The following criteria were used to quantify behavioural performance: 10 trials per level of resistance were analysed in the pre-perturbation phase, and 30-40 trials in the post-perturbation phase. The first trial after the change of resistance level to the opening within the same session was removed, as the resistance was not predictable in this particular trial in contrast to the subsequent trials at the same level of resistance. To quantify the behavioural data, an automated positioning of the cursors placed to separate the different epochs of the trial or to determine some motor parameters such as maximal grip force was performed, although a manual check of proper placement of cursors was undertaken.

The present analysis was restricted to the four motor parameters mentioned above, thus excluding data related to the load force (needed to counteract the resistance during the opening) as well as the subsequent phases of the trial related to the grasping of the pellets. Nevertheless, the grip force was influenced by the increasing levels of resistance (not only the load force) as the monkey had to progressively increase its grip force on the drawer's knob in order to prevent the fingers from slipping from the knob.

\subsection{3 | Control (sham) cTBS experiments}

To exclude possible external factors that would bias the results, sham-cTBS experiments were performed under exactly 
Resistance level 0
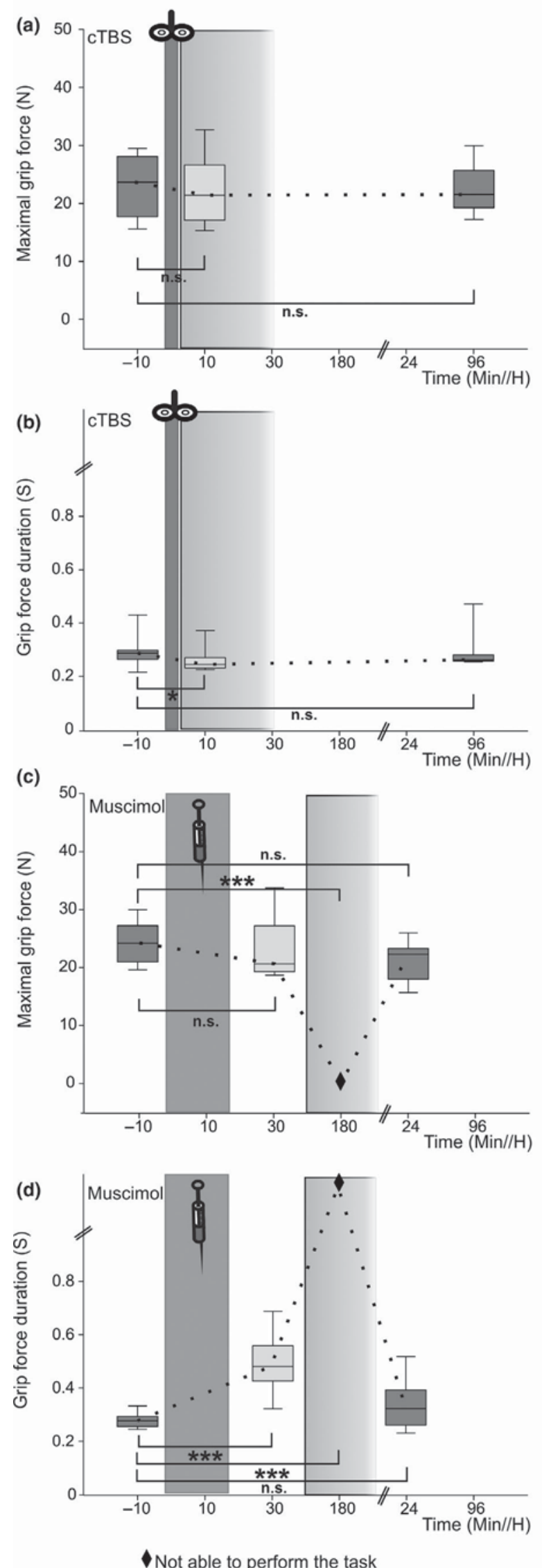

Resistance level 5
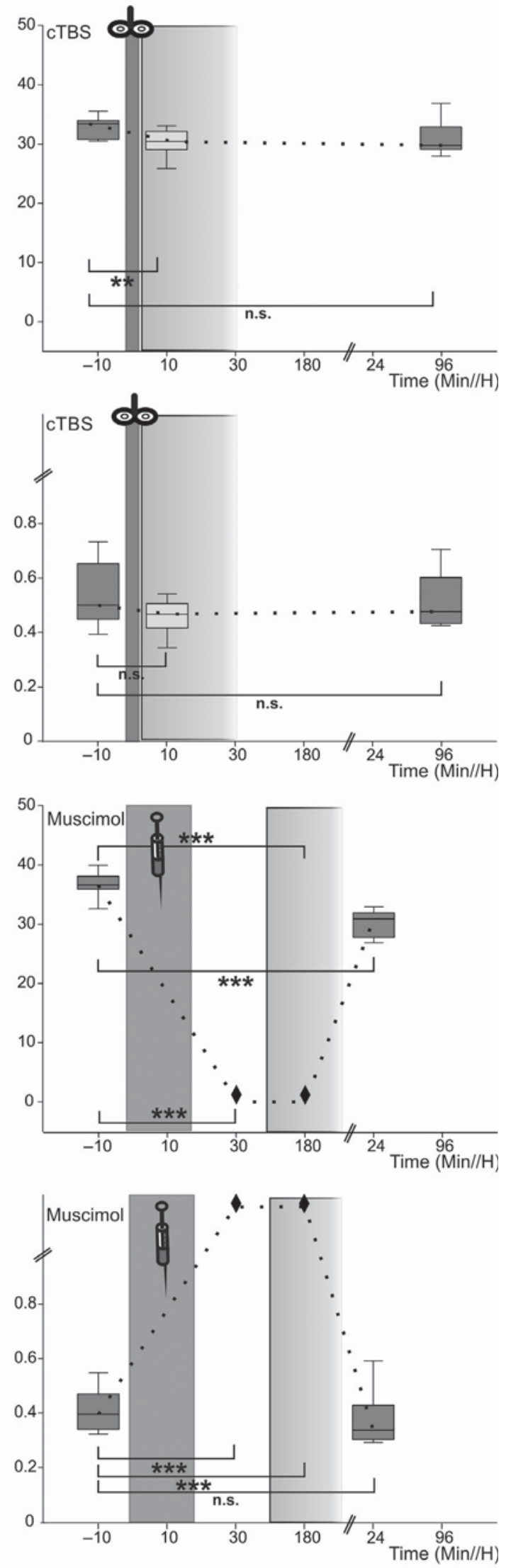
F I G URE 3 Quantification of the grip force exerted on the drawer's knob in order to perform the "reach and grasp" drawer task, illustrated for Mk-CA (hand contralateral to the perturbed/inactivated M1). The data are shown before the transient perturbation (box plot in dark grey), after the perturbation (light grey or diamonds) and in the recovery period (rightmost dark grey box plot). Panels a and b represent effects of cTBS applied onto M1 whereas panels $\mathrm{c}$ and $\mathrm{d}$ are for muscimol infusions in M1. In panels a and c, the maximal grip force was plotted as a function of time (in $\mathrm{min} / \mathrm{hr}$ ). In panels $\mathrm{b}$ and d, the duration of grip force exerted was plotted as a function of time (in $\mathrm{min} / \mathrm{hr}$ ). The results of the statistical comparisons between the different phases of the perturbation experiment are indicated. Diamonds are data points at which the corresponding motor parameter could not be assessed as the monkey was unable to perform the manual dexterity task, due to the strong motor deficit (paresis) resulting from the cortical muscimol inactivation. (***corresponds to a $p$-value $<0.001)$

the same experimental conditions as performed with cTBS (Figure 2a), applying only $1 \%$ of MSO over the corresponding M1 hand area.

\section{6 | Statistics}

As we directly compared the quantified motor parameters in the same animal and within the same experimental session (pre- vs. post-perturbation data), we applied a standard Student's unpaired $t$ test to assess possible differences of the motor parameters before and after cortical inactivation. Levels of statistically significant differences were standard $\left({ }^{*} p\right.$-value $\leq .05, * * p$-value $\leq .01$ and $* * * p$-value $\left.\leq .001\right)$.

\section{\begin{tabular}{l|l}
3 & RESULTS
\end{tabular}}

\section{1 | Characteristics of the "reach and grasp" drawer task}

Although the "reach and grasp" drawer task in this present version has been used in previous studies (Badoud et al., 2017; Borgognon et al., 2019; Kaeser et al., 2014; Schmidlin et al., 2011), it has not been fully validated yet by identifying prominent muscles of the hand and arm involved in the consecutive phases of the task. Chronic recordings of EMG activity were obtained in Mk-CA from eight hand and arm muscles during the performance of the "reach and grasp" drawer task (Figure 1c). Proximal muscles such as the anterior part of the deltoid and the triceps showed an increased EMG activity in the reaching phase of the task. Extrinsic hand muscles such as the flexor digitorum superficialis (FDS), the flexor carpi ulnaris (FCU), the palmaris longus (PL), the extensor digitorum communis (EDC), and two intrinsic hand muscles, the abductor pollicis brevis (AbPB) and the one dorsal interosseous (1DI), were directly involved in the precision grip. The two intrinsic hand muscles showed an increased EMG activity already in the pre-shaping phase, $200-300 \mathrm{~ms}$ before the hand touched the knob of the drawer and, as expected, more pronounced during the grasping phase. Meanwhile, no EMG increase was observed in the pre-shaping phase, when the relative resistance to the opening was increased $(\mathrm{R}=0-5)$ whereas a significant $20 \%$ increase of maximal EMG activity of the AbPB was measured. Figure 1c also illustrates the muscular activity changes as a function of the drawer resistance. For instance, at R5 not only the hand muscles (1DI and AbPB) but also the proximal anterior deltoid showed a strongly increased EMG activity over time immediately after the "knob touch" until full drawer opening, as compared to R0.

The quantified motor parameters of manual dexterity during the "reach and grasp" drawer task were divided into two majors groups. First, the ability of the animal to hold the drawer's knob between the index finger and the thumb generating a continuously measured grip force (Figure 1c), from which the maximal grip force and its duration were derived. Second, two different time epochs of the task, namely the "opening delay time" and the "pulling time." In each successful trial, when the fingers touched the knob, then, there was a progressive increase of grip force exerted between the index finger and the thumb, reaching a maximal value. Together with the load force (not analysed here), the maximal grip force is needed to overcome the level of resistance to the opening of the drawer and thus keep contact with the drawer's knob. In the pre-perturbation periods, as expected, the maximal grip force increased as a function of the resistance to the opening (Figures 1c and 3a,c). In parallel, the grip force duration increased as well as a function of resistance to the opening (Figures $1 \mathrm{c}$ and $3 \mathrm{~b}, \mathrm{~d}$ ).

\section{2 | Pre-perturbation data}

The inactivation procedures, either with cTBS or muscimol, were conducted in the left hemisphere in Mk-CA (left M1) whereas they were applied to the right M1 in Mk-DG (Figure 2a). Typical pre-perturbation trials of the "reach and grasp" drawer task are shown for Mk-CA at resistance levels R0 (Video S1) and R5 (Video S2). The assessment of the grip force yielded comparable results in the pre-perturbation phase of both muscimol cortical inactivation and cTBS cortical perturbation experiments, including the dependence on the level of resistance to the opening (Figure 3; leftmost box plot in each graph). This has been observed for both the maximal grip force (Figure 3a,c) and the grip force duration (Figure 3b,d). Similarly, the pre-perturbation data were also comparable for the two quantified epochs of the task, namely the opening delay time and the pulling time (Figure 4; leftmost box plot in each graph). 

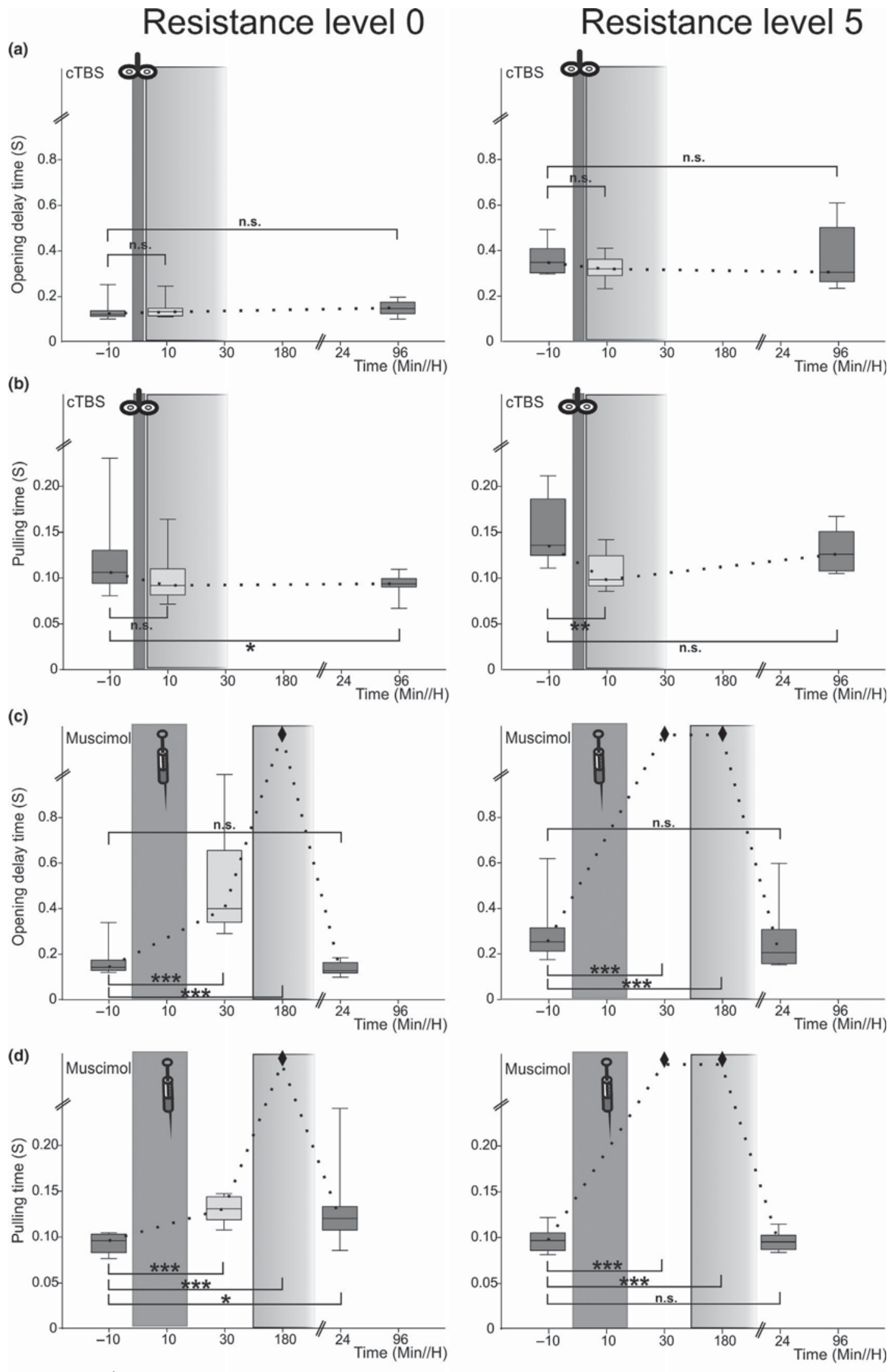

Not able to perform the task

F IG U RE 4 Same as Figure 3, but for the two different time intervals taken into consideration, namely the opening delay time and the pulling (in ordinates) plotted as a function of time ( $\mathrm{min} / / \mathrm{hr}$ ). (*corresponds to a $p$-value $<0.05$, ** $p$-value $<0.01$ and $* * * p$-value $<0.001)$. Same conventions as in Figure 3 


\section{3 | cTBS perturbation}

The effects of the cTBS perturbation on motor behaviour were tested $10 \mathrm{~min}$ after the cTBS (Figure 2b), a time point at which the effects are expected to be at their maximum (Goldsworthy et al., 2012; Suppa et al., 2016), as well as 2-3 days (96 hr) later, when full motor function recovery took place (Figures 3 and 4). Typical "reach and grasp" trials at those time points are illustrated for Mk-CA in Videos S3, S4, S6 and S7. In Mk-CA, there were overall four individual daily cTBS sessions, interspersed with three sham-cTBS sessions (Figure 2a and Table 1). The cTBS session with the maximal behavioural effect obtained in Mk-CA is illustrated in detail in Figures 3 and 4 (panels a and b) and in Table 1 (symbol \#). In Mk-DG, two cTBS sessions took place, preceded by a sham-cTBS session (Figure $2 \mathrm{a}$ ).

As shown for Mk-CA for a typical cTBS session in Figures 3 and 4 (panels a and b) and in Table 1 (symbol \#), consistent with an inhibitory effect of cTBS, there was a decrease of maximal grip force, significant at resistance level R5, but not significant at R0. As far as the temporal parameters are concerned, cTBS induced a decrease of the grip force duration (statistically significant at R0 only), an increase (at R0) and a decrease (at R5) of opening delay time (both nonsignificant though), and a decrease of pulling time (significant at R5 only). Three days after cTBS (see Videos S6 and $\mathrm{S} 7$ ), the vast majority of motor parameters fully recovered, as they did not show significant difference with the pre-cTBS data (Figures 3 and 4, panels a and b). Two more identical daily cTBS sessions were conducted in Mk-CA, whose results are listed in Table 1 (rows Mk-CA 2 and 3 from top). In one of them (second row in Table 1), the data show that all deviations from the pre-perturbation state go in the very same direction as in the previous session mentioned above (symbol \# in Table 1). However, there are differences between the two sessions in the amplitude of these deviations and, moreover, only the maximal grip force decreases at R0 and R5 were statistically significant. The third daily cTBS session in MkCA (third row in Table 1) illustrates the variability of the effects, as for some parameters deviations were in the opposite directions as compared to the other two sessions, on top to some strong variations in the amplitude of the effects. Finally, still in Mk-CA, a last session was conducted, consisting in two consecutive cTBS exposures $15 \mathrm{~min}$ apart (Figure 2a; Table 1, 4th row from top). The data were largely consistent (direction wise) with the single cTBS session which produced the strongest effect (symbol \# in Table 1), but again, there were differences in the amplitude of the changes (with a trend to be slightly larger in case of double cTBS). This indicates that the extra cTBS exposure in the double paradigm did not perturb dramatically more the motor behaviour, except that the decreases of opening delay time and pulling time were statistically more significant at resistance level R5.
The two cTBS sessions in Mk-DG (Table 1) also yielded moderate and variable effects. In the first cTBS session, there was a statistically significant decrease of maximal grip force at R0 but not at R5 (slight increase). In the second session, the maximal grip force decreased at both resistances, though without statistically significant differences. The modest effects were consistent between the two cTBS sessions in Mk-DG as far as the duration of grip force and the opening delay time were concerned. Finally, variable effects were observed for the pulling time at the resistance R5 (Table 1). In both monkeys, the sham-cTBS sessions (Figure 2a) did not induce any behavioural change related to the "reach and grasp" drawer task, as expected.

In summary, the cTBS generated modest to moderate effects on the motor behaviour of the contralateral hand, with changes consistent with an inhibition in most cases for the grip force (amplitude and duration), though statistically significant in only one-third of the cases (Table 1). The effects on the time intervals (opening delay time and pulling time) were more mixed (inhibition/longer interval vs. facilitation/ shorter interval), with however a predominance of shorter intervals (Table 1). A general decrease of the time intervals measured in the manual dexterity task was not expected.

\section{4 $\mid$ Muscimol inactivation}

Muscimol intracortical microinfusion led to pronounced changes of motor parameters in the contralateral hand $30 \mathrm{~min}$ already after the last infusion (Table 1, data in brackets; Figures $3 \mathrm{c}, \mathrm{d}$ and $4 \mathrm{c}, \mathrm{d}$ ), as both animals were unable to perform the task at the highest level of resistance to the opening (R5; see Video S4). At the lowest level of resistance (R0; see Video S3), there was a slight decrease of maximal grip force in Mk-CA (though significant in Mk-DG), accompanied by significant increases of grip force duration, opening delay time and pulling time in both monkeys. Three hours after the muscimol inactivation, a time point at which muscimol is highly potent, there was a complete inability for both animals to perform the task (see Video S5). This is true at both levels of resistance to the opening of the drawer (Figures 3c,d ans $4 \mathrm{c}$,d; grip force amplitude depicted with "nil" and time intervals depicted with diamonds in Table 1). In Mk-CA, the motor ability almost completely recovered from pharmacological inactivation $24 \mathrm{hr}$ after the muscimol infusions (Figures 3 and 4; Videos S6 and S7); the exceptions are the maximal grip force at resistance level 5 and the pulling time at resistance level 0 , with incomplete recovery. As mentioned in the methods, the muscimol inactivation in Mk-DG was long lasting, due to overdosing. In summary, intracortical infusion of muscimol in M1 provoked a dramatic behavioural effect on the contralateral hand, as the monkeys were unable to produce enough force to maintain the drawer's knob in between the thumb and index finger to perform the subsequent pulling action. 


\section{DISCUSSION}

The hypothesis that M1 inactivation with cTBS or muscimol infusion produces comparable impairments of manual dexterity, though to a different extent, was not fully verified. On one hand, different extents of perturbation were observed as expected, with a clearly more dramatic impact of the pharmacological block, corresponding to a local invasive cortical inactivation, whereas cTBS provoked a much lighter perturbation. On the other hand, the behavioural impairments were not consistent in terms of direction of changes between the two methods. Variable and inconsistent effects were observed with cTBS whereas muscimol provoked a strong decrease of grip force and an increase in task duration, as expected. Indeed, muscimol infused in M1 provoked a nearly complete paresis of the contralateral hand and therefore an incapacity to perform the "reach and gasp" drawer task. In contrast, cTBS affected only moderately if not modestly the maximal grip force (decreased as expected in most cases). Furthermore, the changes observed on the temporal characteristics of the task were inconsistent and, in most cases, not in line with a clear inhibitory effect (decrease instead of increase of time intervals), despite the post-perturbation control of M1 localization using single pulse TMS. From these data, it can be concluded that cTBS exerts clearly more moderate effects, not consistent with a pure cortical inhibition whereas muscimol inactivation exerts a potent cortical inhibition strongly influencing the motor behaviour. One noticeable difference between both animals involved in this study is the extended time of functional recovery observed in Mk-DG after the muscimol inactivation, due to an increased concentration of muscimol (see Methods 2). A further difference between these two monkeys is that Mk-DG, for the purpose of the main project (Savidan et al., 2017), was subjected to a permanent M1 lesion in the opposite hemisphere (left M1), produced by ibotenic acid infusion which took place in between the cTBS session and the muscimol session (Figure 2a). However, the muscimol session in Mk-DG (right M1) occurred 4 months after the permanent lesion of the left M1, a time point at which the functional recovery of the right hand reached a plateau (Savidan et al., 2017). In other words, the overdosed muscimol session took place when Mk-DG was in a stable behavioural state regarding its affected right hand, while the subsequent muscimol session in the right M1 challenged the left hand, only marginally affected by the permanent lesion of the left M1 4 months earlier (Savidan et al., 2017). The muscimol session conducted in Mk-DG was most likely not affected by substantial on-going plastic rearrangements post-lesion, which took place earlier, suggesting that the muscimol session in Mk-DG was largely comparable to a session conducted in the intact state. The overdose, however, with its longer lasting effects and some residual deficits after a few weeks, confirm that the standard concentration of $1 \mu \mathrm{g} /$ $\mu \mathrm{l}$ is indeed optimal to obtain a strong effect and a complete functional recovery in 1 day. Higher concentrations (e.g., five times more) may compromise the complete recovery as shown here by Mk-DG. The present muscimol inactivation sessions in Mk-CA and Mk-DG both emphasize the importance of the time course. After $30 \mathrm{~min}$, there were substantial effects already, but they were clearly more pronounced $3 \mathrm{hr}$ after the offset of infusion. In a previous study (Rouiller et al., 1997), reversible pharmacological inactivation was obtained with infusion of lidocaine, with a quicker effect observed after 5 min already, while a substantial recovery occurred also rapidly, 30 min post-infusion. Although the tested tasks were different (modified Brinkman board vs. "reach and grasp" drawer task), muscimol is more potent and longer lasting in order to reversibly inactivate a motor cortical area.

\subsection{Comparison with previous studies}

As compared to previous assessments of manual dexterity in non-human primates based mostly on motor scores either in the modified Brinkman board task or in the Klüver board task (e.g., Liu \& Rouiller, 1999; Kaeser et al., 2010; Hoogewoud et al., 2013; Kaeser et al., 2013; Wyss et al., 2013; Savidan et al., 2017), the "reach and grasp" drawer task is an adequate behavioural task to challenge manual dexterity. The advantage of the "reach and grasp" drawer task is that it offers precise measurements of the force exerted by the monkey, a parameter more sensitive than scoring to assess the effects of cortical perturbation, although force and scoring remain complementary assessments of manual dexterity.

Microinfusion of muscimol in functionally identified M1 hand representation resulted in a strong impairment of the contralateral hand (Figures 3 and 4), in line with previously reported studies using the same or parent (lidocaine) inactivation methods, but for other motor tasks (Kermadi et al., 1997; Liu \& Rouiller, 1999; Murata et al., 2015; Rouiller et al., 1997; Schmidlin et al., 2008). As a control, note that no behavioural effects were observed when physiological saline solution was infused in M1, either in the intact monkey (Kermadi et al., 1997; Rouiller et al., 1997) or after lesion of the contralateral M1 (Liu \& Rouiller, 1999), a control experiment therefore not repeated here. In sharp contrast to muscimol inactivation, cTBS induced only moderate effects on manual dexterity, corresponding to a slight impairment of force production but, surprisingly, a facilitation in terms of temporal sequence of the task. To the best of our knowledge, the present study is original as it is the first one to directly compare in non-human primates, the muscimol and the cTBS perturbation methods for the very same motor task in the same subject. So far, most cTBS studies in animal models were conducted in rodents (Suppa et al., 2016 for review), with the limitation of the small brain size of the rat or, even more so, of the mouse. With that respect, although the monkey brain remains smaller than the human brain, 
its size in macaques still allows a fairly selective cTBS effect on a given brain area such as M1 hand area. The cTBS rodent data showed a large variability, more pronounced inter-individually than intra-individually (Suppa et al., 2016 for review). Although limited to two monkeys, the cTBS variability also appeared between $\mathrm{Mk}-\mathrm{CA}$ and $\mathrm{Mk}-\mathrm{DG}$. This variability was also present intra-individually in $\mathrm{Mk}-\mathrm{CA}$ in which four cTBS sessions took place, with variable and somewhat inconsistent results. Clearly, muscimol inactivation exhibited much less variability than cTBS, both interand intra-individually.

In a study performed in macaque monkeys (Gerits et al., 2011), the authors observed consistent effects of cTBS on the function of the frontal eye field (FEF) in saccadic movements of the eyes, namely a decreased saccadic reaction time. These cTBS data were compared with an earlier study based on reversible inactivation of the FEF using microinfusion of muscimol in NHP (Dias \& Segraves, 1999), in which the inactivation resulted in two effects: an increased latency of the saccades, in parallel with larger targeting errors. In our study, in which the animals were each subjected to both types of inactivation, we observed largely comparable results, namely a decreased pulling time at high level of resistance to the opening in the cTBS paradigm, and a complete inability to perform the task in the muscimol paradigm. The diverging results obtained in the two different types of inactivation of the FEF were attributed to differences of excitability of functionally distinct subpopulations of neurons (Gerits et al., 2011).

The reasons for a very limited and variable effect of cTBS on the motor behaviour as observed here in the macaque monkey remain unknown, in the absence of simultaneous electrophysiological recording in M1 while cTBS is applied. Such simultaneous electrophysiological recordings were performed in the macaque, not for cTBS but for TMS generating an activation of the corresponding cortical area (Bungert, Antunes, Espenhahn, \& Thielscher, 2016; Mueller et al., 2014). Whereas muscimol induces most likely a strong and wide block of most neurons in the targeted cortical area across all layers, thus including the pyramidal neurons at the origin of the corticospinal projection (cortico-motoneuronal in primates), cTBS generates more subtle effect, most likely affecting predominantly superficial cortical layers, in the form of a mix of LTP and LTD plasticity, though with a predominance of inhibitory effects (Suppa et al., 2016). It may be that the effect of cTBS is limited on the indirect corticospinal waves whereas muscimol may affect both the direct and indirect waves (Shimazu et al., 2004; Suppa et al., 2016). With variable effects over the motor cortex, also depending on its excitation level during voluntary contraction (Todd, Rogasch, Flavel, \& Ridding, 2009), cTBS may still induce changes of cortical excitability, but they seem to be not potent enough to sufficiently depress the primary motor cortex and thus do not significantly impact on the motor behaviour, such as manual dexterity in the macaques.

\section{CONCLUSION}

Although cTBS modifies cortical excitability in M1 in macaque monkeys, the behavioural consequences appear modest to moderate at most, with substantial variability, in contrast to the strong and reliable effect obtained by pharmacological inactivation. Consequently, cTBS is a useful tool to induce cortical perturbation, but up to a limited extent, clearly not corresponding to a significant inhibition of the targeted cortical area, as obtained with pharmacological block.

\section{ACKNOWLEDGEMENTS}

This study could not have been completed without the precious help of Laurent Bossy, Jacques Maillard and Josef Corpataux, our professional animal care takers, Veronique Moret (videos), Laurent Monney (informatics), André Gaillard (mechanics), Andrea Francovich and Bernard Aebischer (electronics) and Christine Roulin (technician). We also want to thank Dr. A.-D. Gindrat for her valuable scientific advises, and Dr. P. De Luna, Dr. M. Mouthon and Dr. M. Faiz Bin Mustafar for their technical help. This work was funded by the Grant PZ00P3_142258 (E.S.) and the grants 31-61857.00, 310000-110005, 31003A-132465 and 31003A-149643 (E.M.R.) from the Swiss National Foundation for Research. The present study was supported by the Swiss Primate Competence Center for Research (SPCCR: see www.unifr.ch/spccr/).

\section{CONFLICT OF INTEREST}

The authors declare to have no conflict of interest in relation to the present study.

\section{AUTHORS' CONTRIBUTIONS}

EMR and ES designed the study. CR, MK, JS and ES performed the experiments. CR, MK, JS, MF and ES analysed the data. CR and ES drafted the article. EMR and ES finalized and revised the article.

\section{DATA ACCESSIBILITY}

The behavioural, electrophysiological and histological data are publicly available on request to the corresponding author.

\section{ORCID}

Eric M. Rouiller (iD https://orcid.org/0000-0003-1355-6019 Eric Schmidlin (iD https://orcid.org/0000-0001-8638-2369 


\section{REFERENCES}

Andersen, P., Hagan, P. J., Phillips, C. G., \& Powell, T. P. (1975). Mapping by microstimulation of overlapping projections from area 4 to motor units of the baboon's hand. Proceedings of the Royal Society of London. Series B: Biological Sciences, 188, 31-36.

Badoud, S., Borgognon, S., Cottet, J., Chatagny, P., Moret, V., Fregosi, M., ... Rouiller, E. M. (2017). Effects of dorsolateral prefrontal cortex lesion on motor habit and performance assessed with manual grasping and control of force in macaque monkeys. Brain Structure and Function, 222, 1193-1206. https://doi.org/10.1007/ s00429-016-1268-z

Bestmann, S., Swayne, O., Blankenburg, F., Ruff, C. C., Teo, J., Weiskopf, N., ... Ward, N. S. (2010). The role of contralesional dorsal premotor cortex after stroke as studied with concurrent TMSfMRI. The Journal of Neuroscience, 30, 11926-11937. https://doi. org/10.1523/JNEUROSCI.5642-09.2010

Borgognon, S., Cottet, J., Moret, V., Chatagny, P., Carrara, L., Fregosi, M., ... Badoud, S. (2019). Fine manual dexterity assessment after autologous neural cell ecosystems (ANCE) transplantation INA nonhuman primate model of Parkinson's disease. Neurorehabilitation and Neural Repair, 33, 553-567. https://doi.org/10.1177/15459 68319850133

Brochier, T., Boudreau, M. J., Pare, M., \& Smith, A. M. (1999). The effects of muscimol inactivation of small regions of motor and somatosensory cortex on independent finger movements and force control in the precision grip. Experimental Brain Research, 128, 31-40. https://doi.org/10.1007/s002210050814

Bungert, A., Antunes, A., Espenhahn, S., \& Thielscher, A. (2016). Where does TMS Stimulate the Motor Cortex? Combining electrophysiological measurements and realistic field estimates to reveal the affected cortex position. Cerebral Cortex, 27, 5083-5094.

Contestabile, A., Colangiulo, R., Lucchini, M., Gindrat, A. D., Hamadjida, A., Kaeser, M., ... Schmidlin, E. (2018). Asymmetric and distant effects of a unilateral lesion of the primary motor cortex on the bilateral supplementary motor areas in adult macaque monkeys. The Journal of Neuroscience, 38, 10644-10656. https://doi. org/10.1523/JNEUROSCI.0904-18.2018

Dancause, N., Barbay, S., Frost, S. B., Plautz, E. J., Chen, D., Zoubina, E. V., ... Nudo, R. J. (2005). Extensive cortical rewiring after brain injury. The Journal of Neuroscience, 25, 10167-10179. https://doi. org/10.1523/JNEUROSCI.3256-05.2005

Darling, W. G., Pizzimenti, M. A., Rotella, D. L., Hynes, S. M., Ge, J., Stilwell-Morecraft, K., \& Morecraft, R. J. (2016). Sensorimotor cortex injury effects on recovery of contralesional dexterous movements in Macaca mulatta. Experimental Neurology, 281, 37-52. https://doi.org/10.1016/j.expneurol.2016.04.004

De Luna, P., Mohamed Mustafar, M. F., \& Rainer, G. (2014). A MATLAB-based eye tracking control system using non-invasive helmet head restraint in the macaque. Journal of Neuroscience Methods, 235, 41-50. https://doi.org/10.1016/j.jneumeth.2014.05.033

Dias, E. C., \& Segraves, M. A. (1999). Muscimol-induced inactivation of monkey frontal eye field: Effects on visually and memory-guided saccades. Journal of Neurophysiology, 81, 2191-2214. https://doi. org/10.1152/jn.1999.81.5.2191

Finger, S. (2010). Chapter 51: Recovery of function: Redundancy and vicariation theories. Handbook of Clinical Neurology, 95, 833-841.

Georgiev, D., Rocchi, L., Tocco, P., Speekenbrink, M., Rothwell, J. C., \& Jahanshahi, M. (2016). Continuous theta burst stimulation over the dorsolateral prefrontal cortex and the Pre-SMA alter drift rate and response thresholds respectively during perceptual decisionmaking. Brain Stimulation, 9, 601-608. https://doi.org/10.1016/j. brs.2016.04.004

Gerits, A., Ruff, C. C., Guipponi, O., Wenderoth, N., Driver, J., \& Vanduffel, W. (2011). Transcranial magnetic stimulation of macaque frontal eye fields decreases saccadic reaction time. Experimental Brain Research, 212, 143-152. https://doi.org/10.1007/ s00221-011-2710-3

Goldsworthy, M. R., Pitcher, J. B., \& Ridding, M. C. (2012). A comparison of two different continuous theta burst stimulation paradigms applied to the human primary motor cortex. Clinical Neurophysiology, 123, 2256-2263. https://doi.org/10.1016/j. clinph.2012.05.001

Hoogewoud, F., Hamadjida, A., Wyss, A. F., Mir, A., Schwab, M. E., Belhaj-Saif, A., \& Rouiller, E. M. (2013). Comparison of functional recovery of manual dexterity after unilateral spinal cord lesion or motor cortex lesion in adult macaque monkeys. Frontiers in Neurology, 4, 101.

Huang, Y. Z., Edwards, M. J., Rounis, E., Bhatia, K. P., \& Rothwell, J. C. (2005). Theta burst stimulation of the human motor cortex. Neuron, 45, 201-206. https://doi.org/10.1016/j.neuron.2004.12.033

Kaeser, M., Chatagny, P., Gindrat, A. D., Savidan, J., Badoud, S., Fregosi, M., ... Rouiller, E. M. (2014). Variability of manual dexterity performance in non-human primates (Macaca fascicularis). International Journal of Comparative Psychology, 27, 295-325.

Kaeser, M., Wannier, T., Brunet, J. F., Wyss, A., Bloch, J., \& Rouiller, E. M. (2013). Representation of motor habit in a sequence of repetitive reach and grasp movements performed by macaque monkeys: Evidence for a contribution of the dorsolateral prefrontal cortex. Cortex, 49, 1404-1419. https://doi.org/10.1016/j. cortex.2012.05.025

Kaeser, M., Wyss, A. F., Bashir, S., Hamadjida, A., Liu, Y., Bloch, J., ... Rouiller, E. M. (2010). Effects of unilateral motor cortex lesion on ipsilesional hand's reach and grasp performance in monkeys: Relationship with recovery in the contralesional hand. Journal of Neurophysiology, 103, 1630-1645. https://doi.org/10.1152/ jn.00459.2009

Kazennikov, O., Wicki, U., Corboz, M., Hyland, B., Palmeri, A., Rouiller, E. M., \& Wiesendanger, M. (1994). Temporal structure of a bimanual goal-directed movement sequence in monkeys. European Journal of Neuroscience, 6, 203-210. https://doi. org/10.1111/j.1460-9568.1994.tb00262.x

Kermadi, I., Liu, Y., Tempini, A., \& Rouiller, E. M. (1997). Effects of reversible inactivation of the supplementary motor area (SMA) on unimanual grasp and bimanual pull and grasp performance in monkeys. Somatosensory and Motor Research, 14, 268-280. https://doi. org/10.1080/08990229770980

Lanz, F., Lanz, X., Scherly, A., Moret, V., Gaillard, A., Gruner, P., ... Rouiller, E. M. (2013). Refined methodology for implantation of a head fixation device and chronic recording chambers in non-human primates. Journal of Neuroscience Methods, 219, 262-270. https:// doi.org/10.1016/j.jneumeth.2013.07.015

Lawrence, D. G., Porter, R., \& Redman, S. J. (1985). Corticomotoneuronal synapses in the monkey: Light microscopic localization upon motoneurons of intrinsic muscles of the hand. The Journal of Comparative Neurology, 232, 499-510. https://doi.org/10.1002/(ISSN)1096-9861

Lemon, R. N. (2008a). Descending pathways in motor control. Annual Review of Neuroscience, 31, 195-218. https://doi.org/10.1146/annur ev.neuro.31.060407.125547 
Lemon, R. N. (2008b). An enduring map of the motor cortex. Experimental Physiology, 93, 798-802. https://doi.org/10.1113/ expphysiol.2007.039081

Lemon, R. N., \& Griffiths, J. (2005). Comparing the function of the corticospinal system in different species: Organizational differences for motor specialization? Muscle and Nerve, 32, 261-279. https:// doi.org/10.1002/(ISSN)1097-4598

Liu, Y., \& Rouiller, E. M. (1999). Mechanisms of recovery of dexterity following unilateral lesion of the sensorimotor cortex in adult monkeys. Experimental Brain Research, 128, 149-159. https://doi. org/10.1007/s002210050830

Morecraft, R. J., Ge, J., Stilwell-Morecraft, K. S., McNeal, D. W., Hynes, S. M., Pizzimenti, M. A., ... Darling, W. G. (2016). Frontal and frontoparietal injury differentially affect the ipsilateral corticospinal projection from the nonlesioned hemisphere in monkey (Macaca mulatta). The Journal of Comparative Neurology, 524, 380-407. https://doi.org/10.1002/cne.23861

Mueller, J. K., Grigsby, E. M., Prevosto, V., Petraglia 3rd, F. W., Rao, H., Deng, Z. D., ... Grill, W. M. (2014). Simultaneous transcranial magnetic stimulation and single-neuron recording in alert nonhuman primates. Nature Neuroscience, 17, 1130-1136. https://doi. org/10.1038/nn.3751

Murata, Y., Higo, N., Hayashi, T., Nishimura, Y., Sugiyama, Y., Oishi, T., ... Onoe, H. (2015). Temporal plasticity involved in recovery from manual dexterity deficit after motor cortex lesion in macaque monkeys. The Journal of Neuroscience, 35, 84-95. https://doi. org/10.1523/JNEUROSCI.1737-14.2015

Nyffeler, T., Cazzoli, D., Hess, C. W., \& Muri, R. M. (2009). One session of repeated parietal theta burst stimulation trains induces longlasting improvement of visual neglect. Stroke, 40, 2791-2796. https ://doi.org/10.1161/STROKEAHA.109.552323

Rouiller, E. M., Yu, X. H., \& Tempini, A. (1997). Effect of inactivation of the hand representation of the primairy and supplementary motor cortical areas on preicsion grip performance in monkeys. In M. C. Hepp-Reymond, \& G. Marini (Eds.), Perspectives of motor behavior and its neural basis (pp. 33-43). Basel, Switzerland: KArger. https ://doi.org/10.1159/000062750

Rouse, A. G., \& Schieber, M. H. (2016). Spatiotemporal distribution of location and object effects in primary motor cortex neurons during reach-to-grasp. The Journal of Neuroscience, 36, 10640-10653. https://doi.org/10.1523/JNEUROSCI.1716-16.2016

Savidan, J., Kaeser, M., Belhaj-Saif, A., Schmidlin, E., \& Rouiller, E. M. (2017). Role of primary motor cortex in the control of manual dexterity assessed via sequential bilateral lesion in the adult macaque monkey: A case study. Neuroscience, 357, 303-324. https:// doi.org/10.1016/j.neuroscience.2017.06.018

Schaechter, J. D., Fricker, Z. P., Perdue, K. L., Helmer, K. G., Vangel, M. G., Greve, D. N., \& Makris, N. (2009). Microstructural status of ipsilesional and contralesional corticospinal tract correlates with motor skill in chronic stroke patients. Human Brain Mapping, 30, 3461-3474. https://doi.org/10.1002/hbm.20770

Schmidlin, E., Brochier, T., Maier, M. A., Kirkwood, P. A., \& Lemon, R. N. (2008). Pronounced reduction of digit motor responses evoked from macaque ventral premotor cortex after reversible inactivation of the primary motor cortex hand area. The Journal of Neuroscience, 28, 5772-5783. https://doi.org/10.1523/JNEUROSCI.0944-08.2008
Schmidlin, E., Kaeser, M., Gindrat, A. D., Savidan, J., Chatagny, P., Badoud, S., ... Rouiller, E. M. (2011). Behavioral assessment of manual dexterity in non-human primates. Journal of Visualized Experiments, 57, e3258.

Schmidlin, E., Wannier, T., Bloch, J., \& Rouiller, E. M. (2004). Progressive plastic changes in the hand representation of the primary motor cortex parallel incomplete recovery from a unilateral section of the corticospinal tract at cervical level in monkeys. Brain Research, 1017, 172-183. https://doi.org/10.1016/j.brain res.2004.05.036

Shimazu, H., Maier, M. A., Cerri, G., Kirkwood, P. A., \& Lemon, R. N. (2004). Macaque ventral premotor cortex exerts powerful facilitation of motor cortex outputs to upper limb motoneurons. The Journal of Neuroscience, 24, 1200-1211. https://doi.org/10.1523/ JNEUROSCI.4731-03.2004

Slavin, M. D., Lawrence, S., \& Stein, D. G. (1998). Another look at vicariation. In S. Finger, T. E. Levere, C. R. Almli, \& D. G. Stein (Eds.), Brain injury and recovery (pp. 165-179). Boston, MA: Springer.

Suppa, A., Huang, Y. Z., Funke, K., Ridding, M. C., Cheeran, B., Di Lazzaro, V., ... Rothwell, J. C. (2016). Ten years of theta burst stimulation in humans: Established knowledge, unknowns and prospects. Brain Stimulation, 9, 323-335. https://doi.org/10.1016/j. brs.2016.01.006

Swayne, O. B., Rothwell, J. C., Ward, N. S., \& Greenwood, R. J. (2008). Stages of motor output reorganization after hemispheric stroke suggested by longitudinal studies of cortical physiology. Cerebral Cortex, 18, 1909-1922. https://doi.org/10.1093/cercor/bhm218

Todd, G., Rogasch, N. C., Flavel, S. C., \& Ridding, M. C. (2009). Voluntary movement and repetitive transcranial magnetic stimulation over human motor cortex. Journal of Applied Physiology, 106, 1593-1603. https://doi.org/10.1152/japplphysiol.91364.2008

Wyss, A. F., Hamadjida, A., Savidan, J., Liu, Y., Bashir, S., Mir, A., ... Belhaj-Saif, A. (2013). Long-term motor cortical map changes following unilateral lesion of the hand representation in the motor cortex in macaque monkeys showing functional recovery of hand functions. Restorative Neurology and Neuroscience, 31, 733-760. 